\title{
Pharmacophore-Based Virtual Screening and Molecular Docking of Approved and Experimental Drugs Against Helicobacter pylori DNA Polymerase III $\beta$-Clamp
}

\section{Prince Messiah G Tan and Junie B Billones*}

Department of Physical Sciences and Mathematics, College of Arts and Sciences, University of The Philippines Manila, Padre Faura, Ermita, Manila, Philippines

*Corresponding Author: Junie B Billones, Department of Physical Sciences and Mathematics, College of Arts and Sciences, University of the Philippines Manila, Padre Faura, Ermita, Manila, Philippines.

Received: November 01, 2019; Published: November 14, 2019

DOI: $10.31080 /$ ASPS.2019.03.0438

\section{Abstract}

The emerging resistance of pathogenic bacteria to present antibiotics is an alarming human health risk worldwide. According to the World Health Organization (WHO), Helicobacter pylori is in the high priority list, and for which new antibiotics should be developed. An interesting drug target in H. pylori is DNA Polymerase III $\beta$-clamp, an enzyme that serves a major role in DNA replication. In this study, the approved and experimental drugs in DrugBank were virtually screened based on a pharmacophore derived from the structure of $H$. pylori DNA Pol III $\beta$-clamp. The high-scoring compounds were docked and rank-ordered based on their binding energies. The top five drug hits were Benfotiamine, Raltitrexed, Netarsudil, Hydrocortisone succinate, and Glimepiride. The topmost hit, Benfotiamine, possesses most of the characteristics of a good drug, being predicted to be non-carcinogenic, non-mutagenic, nontoxic, and non-inhibitor of cytochrome P540, albeit it has poor solubility, low intestinal absorption, and high plasma protein binding. In general, the other top hit drugs also displayed satisfactory drug properties and can be further developed and repurposed as new antibiotics against $H$. pylori.

Keywords: Helicobacter pylori; Molecular Docking; DNA Polymerase III $\beta$-Clamp; Benfotiamine; Raltitrexed; Netarsudil; Hydrocortisone succinate; Glimepiride

\section{Introduction}

Various type of pathogens have perennially afflicted the human population. In fact, WHO has recently issued a list of bacteria that need to be prioritized due to the emergence of antibiotic resistance. These bacteria were categorized into 12 families according to priority (medium, high, and critical) for drug development [1]. It is noteworthy that Helicobacter pylori (H. pylori) was classified in the high priority group due to its growing resistance primarily to clarithromycin [1,2].

H. pylori is a spiral-rod shaped gram-negative bacterium that lives and survives in a very acidic environment $(\mathrm{pH} \sim 2)$ [3]. The growth of this bacteria in the stomach lining is associated with several human gastric diseases $[4,5]$. The gastric microenvironment of the stomach is hostile to commensal bacteria considering low partial oxygen pressure, high gastric acid concentration, and presence of many digestive enzymes that are, however, beneficial to $H$. pylori. The transmission of $H$. pylori infection occurs via oral-oral or faeco-oral route. Moreover, vertical transmission (i.e. parent to child) is also a common route [5].
The gastric colonization of $H$. pylori results to various human pathological conditions such as peptic ulcer disease, gastric adenocarcinoma, superficial gastritis, etc. These conditions have been managed by combining acid-suppressing medication and antibiotics [5]. However, the emergence of antibiotic-resistant $H$. pylori strains poses a great challenge in the management of bacterial infection associated with $H$. pylori. The eradication rate of about $80 \%$ for the past 20 years with the use of the first-line drug clarithromycin is no longer achieved. This is also true for levofloxacin, an alternative medicine proposed a decade ago [6].

Involved in myriads of steps during prokaryotic replication is a vital protein known as $\beta$-clamp (sliding clamp) that forms a ring shaped dimer encircling the dsDNA. The $\beta$-clamp of $H$. pylori is a homodimer with each monomer consisting of three globular domains yielding a six-domain ring $[7,8]$. In prokaryotes, the cell division rate is high, thus, it is required to maintain the fidelity of DNA replication and repair. For most of the processes, $\beta$-clamp serves as a hub for protein-protein interaction. It also interacts with other proteins including DNA ligase, mismatch repair proteins MutL and 
MutS apart from DNA polymerases [9,10]. The topological state of DNA is the basis of beta-DNA interaction which indicates that the $\beta$-clamp protein binds by virtue of its shape and not by chemical forces. Hence, this $\beta$ subunit acts a mobile sliding clamp tethering Pol III to DNA for high processivity of the synthesis $[8,11]$.

Indeed, the DNA Polymerase III $\beta$-clamp is a highly druggable target in H. pylori. This protein acts a major role in DNA replication and thus in reproduction and survival of the pathogen [12]. There is no reported sequence homology between prokaryotic $\beta$-clamp and eukaryotic PCNA (proliferating cell nuclear antigen) despite of having similar function. Therefore, the use of compounds that target the $\beta$-clamp of $H$. pylori is relatively safe for humans [13].

In this study, we have applied computational approaches in drug discovery that have been proven useful in the rational discovery and development of new drugs $[14,15]$. We have demonstrated in our previous work the utility of in silico techniques in discovering leads against druggable targets in Mycobacterium tuberculosis [1619]. We also found it prudent to explore the drugs database first before moving to a much expansive chemical space. Drug repurposing abbreviates the drug discovery process because the hits or leads have already passed an adequate number of tests on safety and drug-likeness. Hence, we screened the approved and experimental drugs against $H$. pylori DNA Pol III $\beta$-clamp. In particular, a pharmacophore was generated based on the structure of the drug target and the DrugBank database was screened by rigid and flexible fitting procedures. The high-scoring compounds were subsequently docked to DNA Pol III $\beta$-clamp and were rank-ordered based on their binding energies. Furthermore, the relevant pharmacokinetics and pharmacodynamics properties (i.e. ADMET - absorption, distribution, metabolism, excretion, toxicity; carcinogenicity; mutagenicity; etc.) of the top hits were determined in silico.

\section{Materials and Methods}

All computational procedures were done on Accelrys (now Biovia) Discovery Studio (DS) Client v2.5 (https://www.3dsbiovia. com) installed on a computer running on Microsoft Windows 7 Home Premium 64-bit Operating System using an Intel CoreTM i7-4770K CPU, with $8 \mathrm{GHz}$ processor and 16GB random access memory (RAM).

\section{Preparation of H. pylori DNA Pol III $\beta$-clamp}

The crystal structure of $H$. pylori DNA Pol III $\beta$-clamp with 5-chloroisatin ligand (PDB code: 5G4Q) [20] was retrieved from Research Collaboration for Structural Bioinformatics Protein Data Bank (RCSB PDB) (http://www.rcsb.org). The Clean Protein and Prepare Protein tools were used to remove the co-crystallized ligands, water molecules, ions; and to prepare the protein structure. To obtain the lowest energy conformation of the target enzyme, the structure optimization of protein was performed by using the Minimization protocol in DS. The Superimpose protocol was used to align the original protein with the minimized protein to prompt the structures for root-mean-square deviation (RMSD) calculation.

\section{Pharmacophore generation}

The amino acids that were known to interact with the ligand (5-chloroisatin) [20] were selected in defining the binding site with the use of the Define Sphere protocol. The nature of the active site of the target molecule was analyzed for hydrogen bonding and hydrophobic features using the Interaction Generation protocol. The number of features in the initial pharmacophore generated were narrowed down using the Cluster Current Feature and Keep Only Cluster Centers tools.

\section{Virtual screening and molecular docking}

The different tautomers, isomers, ionization states, and 3D conformations were generated for each compound to be screened using the Prepare Ligands protocol. The prepared ligands were converted to 3D structures using the Build 3D Database protocol. The Lipinski Rule of Five was turned off in order to widen the search space and avoid unnecessary exclusion of potential hits. Besides, most of the compounds under consideration, being approved or experimental drugs, have already undergone adequate experimental filters for drug-likeness. In the virtual screening step, rigid and flexible fitting procedures were employed in succession. The Screen Library protocol was used to screen the created 3D database using the generated pharmacophore. The FAST (fluctuation amplification of specific traits) method was used in conformation generation during the virtual screening stage. After screening, the ligands with fit values of 3.0 and above were re-prepared using the Prepare Ligands protocol and were subjected to molecular docking studies. Molecular docking was performed using Dock Ligands (CDOCKER) protocol. The Calculate Binding Energies protocol was used to calculate the binding energies of the ligands with the enzyme target. At this step, in situ minimization and ligand conformation entropy was set to True. The 2D ligand interaction diagrams were generated using Discovery Studio Visualizer (https://www.3dsbiovia. com).

\section{In silico ADME-Tox}

The compounds with stronger binding affinity (i.e. more negative binding energy) than the reference ligand (5-chloroisatin) were subjected to in silico ADME-Tox analysis. The ADMET Descriptors protocol and TOPKAT protocol in DS were used to predict the pharmacodynamics and pharmacokinetics properties of the top hits.

\section{Results and Discussions \\ Validation of prepared protein}

In order to check the effect of protein preparation on the structure of the protein target, the prepared protein structure was su- 
perimposed onto the original crystal structure [20]. It was found that there was only minimal adjustment in the protein structure during preparation as the RMSD was only $0.671 \AA \AA$ (Figure 1 ), a value that is well within the acceptable range $(<1.5 \AA)$ [21].

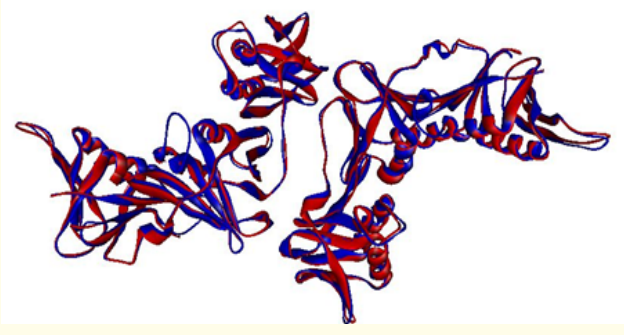

Figure 1: Solid ribbon diagram of the minimized H. pylori DNA Pol III $\beta$-clamp (blue) superimposed to the original H. pylori DNA Pol III $\beta$-clamp protein (red). RMSD $=0.671 \AA$.

\section{Active Site Definition}

The amino acid residues which were known to interact with the co-crystalized ligand, 5-chloroisatin, were used as basis in defining the active site. The generated active site sphere had a radius of 10.0 Å located at the $\mathrm{x}, \mathrm{y}, \mathrm{z}$ coordinates of $-34.9136,11.4405$, 47.3194 , respectively. The active site was specifically positioned at the chain B of the enzyme and contains the following residues: Thr175, Lys176, Arg177, Leu178, and Leu368 (Figure 2).

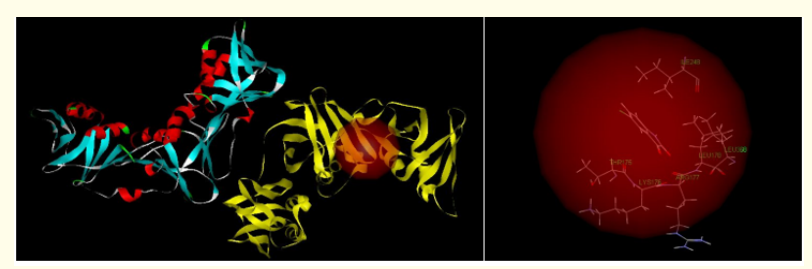

Figure 2: Flat ribbon diagram of the active site location in the protein (left) and the key amino acid residues at the active site (right).

\section{Validation of Docking Method}

The 2D interaction diagram for the original ligand-protein complex (Figure 3a) and the modeled complex (Figure 3b) show that there was no remarkable difference in terms of the amino acid residues involved in the binding. It can be seen that, the conventional hydrogen bond between Thr173 and the amino group of the ligand as well as the carbon H-bond between Arg177 and a carbonyl carbon atom were observed in both complexes. In addition, the van der Waals interactions formed by Asp174, Lys176, and Pro347 were also retained in the resulting model.

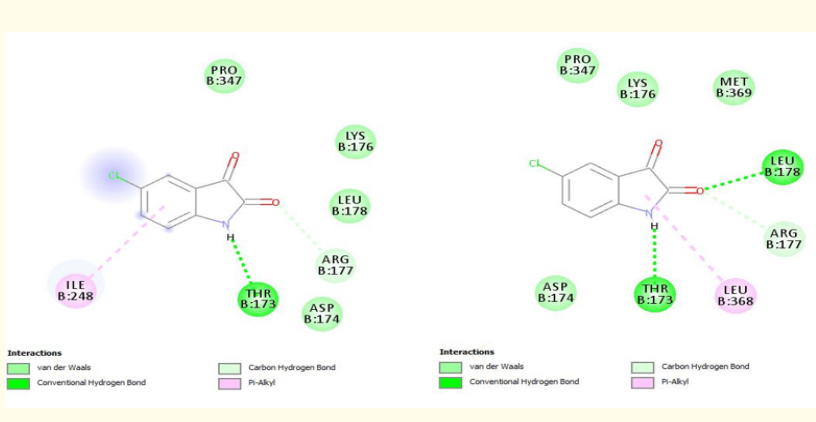

Figure 3: (a) Interaction diagram for 5-chloroisatin in complex with $\beta$-clamp (original crystal structure). (b) Interaction diagram for the redocked 5 -chloroisatin with prepared $\beta$-clamp target.

\section{Pharmacophore generation}

A pharmacophore consists of steric and electronic features that a ligand must possess in order to successfully interact with a particular biological target. Hence, a pharmacophore was generated based on the structure of $H$. pylori DNA Pol III $\beta$-clamp structure. Initially, the pharmacophore consisted of numerous features, but these were subsequently reduced to 30 . Figure 4 shows the type and spatial arrangement of the different features in the generated pharmacophore. The magenta-colored spheres are the hydrogenbond donors, the green spheres are the hydrogen-bond acceptors and blue spheres are the hydrophobes. This 30 -feature pharmacophore was used as basis in the virtual screening of drugs against $H$. pylori DNA Pol III $\beta$-clamp.

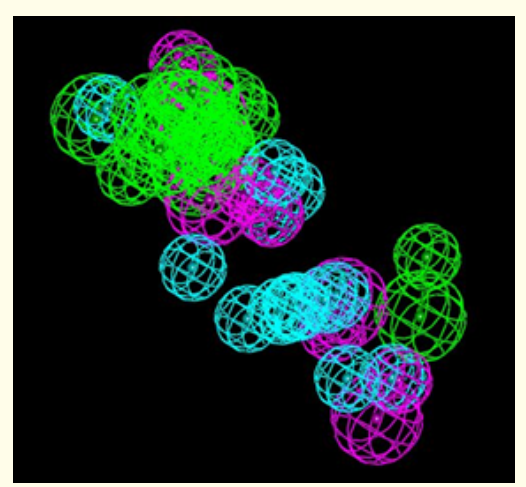

Figure 4: Structure based pharmacophore model with 30 features: 6 donors (magenta), 11 acceptors (green), and 13 hydrophobes (blue).

Virtual screening and molecular docking

The prepared database consisting of approved and experimental drugs was screened based on the pharmacophore generated from the structure of the $\beta$-clamp binding site. The high-scoring compounds (i.e. fit value of least 3.0) were subsequently docked 
to the protein target. Molecular docking was accomplished with the use of the CDOCKER protocol, which employs a molecular dynamics simulated annealing-based algorithm that keeps the protein (enzyme) rigid while letting the ligands to be fully flexible. The resultant docking poses were compared and sorted based on their binding energies. A more negative binding energy value corresponds to a more exergonic complex formation that is indicative of a stronger ligand-protein interaction or more stable complex formation.

The binding energy of the co-crystalized ligand, 5-chloroisatin, was found to be $50.16 \mathrm{kcal} / \mathrm{mol}$. This value served as the reference binding energy with which the binding potential of other ligands docked to $\beta$-clamp was compared. Among the ligands that were subjected to molecular docking, 67 compounds showed more negative binding energies than 5-chloroisatin. These compounds are predicted to have superior inhibitory activity against $H$. pylori DNA Pol III $\beta$-clamp compared to the known ligand.

The binding energies and type of interactions formed by the top 5 hits are detailed in Table 1. Benfotiamine (Accession Number: DB11748) exhibits the most negative binding energy (-241 kcal/ $\mathrm{mol}$ ), which is roughly five times greater than the reference. The next four compounds, namely, raltitrexed, netarsudil, hydrocortisone succinate, and glimepiride, have binding energy values that are at least 4 times larger than that of 5-chloroisatin. In general, the top hits have observably greater number of van der Waals and pialkyl interactions with the target compared to the reference ligand.

\begin{tabular}{|l|c|c|c|c|c|}
\hline Compound & Binding Energy (kcal/mol) & van der Waals & H-bonds & Charged & Pi \\
\hline & -241.38 & 7 & 2 & 1 & 3 \\
\hline & -231.02 & & & \\
\hline \\
\hline Raltitrexed
\end{tabular}

Table 1: Binding Energies and Type of Interactions formed by the top hits and the reference ligand (5-chloroisatin) with Pol III $\beta$-Clamp. 
Figure 5 shows the interaction diagram for the number one top hit. Benfotiamine formed van der Waals interactions with Arg177, Tyr242, Asp244, Tyr245, Leu368 and Met370. It also interacted with the target via conventional hydrogen bonding with Thr173 and Lys176, which simultaneously formed attractive charge interaction with its phosphate group. Moreover, the aromatic rings of Benfotiamine allowed the pi-alkyl interactions to be formed with Leu154, Pro243, and Ile248 residues. Benfotiamine has been developed for the treatment and prevention of Diabetic Nephropathy and Type 2 Diabetes Mellitus [22]. Nevertheless, the results of this work indicate that it can be repurposed as an antibiotic for H. pylori infection particularly as inhibitor of its DNA Pol III $\beta$-clamp.

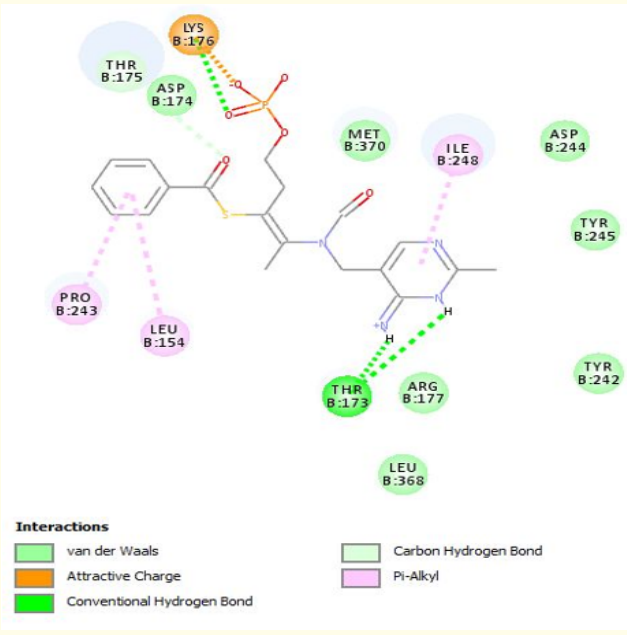

Figure 5: Interaction map for Benfotiamine-Pol III $\beta$-clamp complex.

The second top hit Raltitrexed (Accession Number: DB00293), an approved drug named Tomudex, is an antimetabolite used in cancer chemotherapy. Raltitrexed is an antineoplastic agent and folic acid antagonist. Specifically, it inhibits thymidylate synthase (TS) leading to DNA fragmentation and cell death [12]. Through its hydroxyl group, Raltitrexed formed a hydrogen bond with Thr175, two pi interactions with Ile248 and Leu368, one attractive charge interaction with Lys176, and a host of van der Waals interactions with Met370, Pro243, Arg177, Met369, Leu178, Pro347, Asp174 and Thr173 (Figure 6).

The third top hit netarsudil (Accession Number: DB13931) was quite different. Its interaction with the target was dominated by numerous van der Waals and pi-alkyl interactions (Figure 7). It exhibited only one conventional hydrogen bond with Thr173 and one carbon hydrogen bond with Met370. Netarsudil has been marketed as a novel glaucoma medication that specifically targets the conventional trabecular pathway of aqueous humour outflow. It acts as an inhibitor of rho kinase and norepinephrine transporters
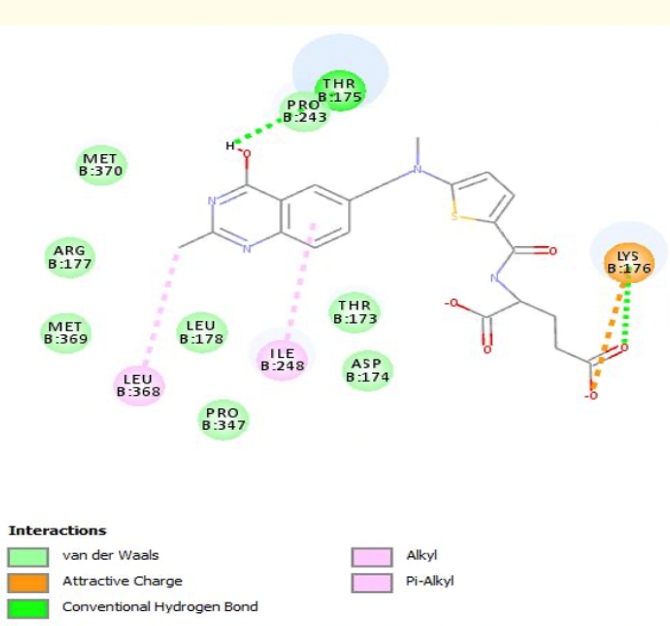

Figure 6: Interaction map for Raltitrexed-Pol III $\beta$-clamp complex.

[23]. However, the results of this work also indicate that netarsudil may also be developed as an antibiotic for $H$. pylori.
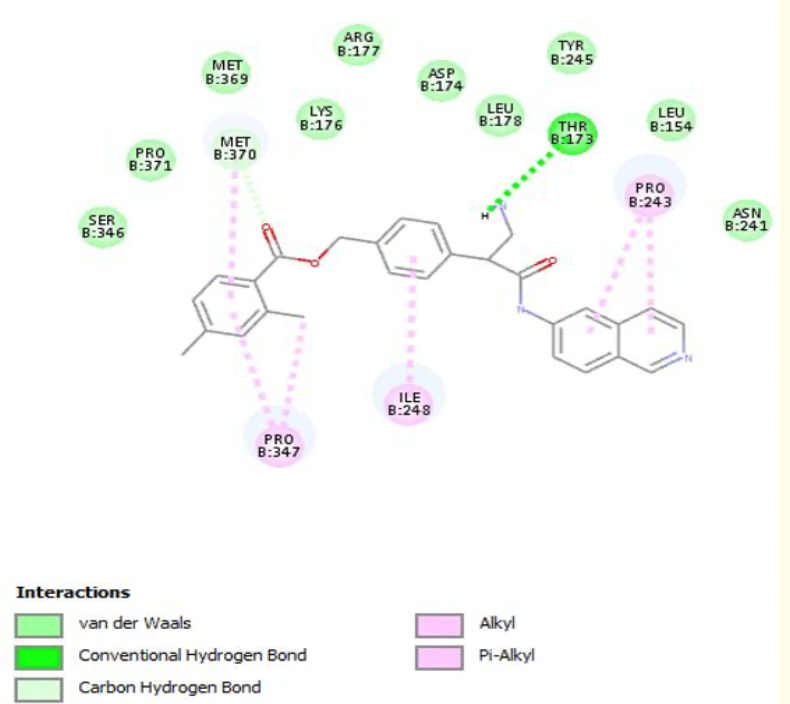

Figure 7: Interaction map for Netarsudil-Pol III $\beta$-clamp complex.

Furthermore, the fourth top hit, hydrocortisone succinate, is an anti-inflammatory and immunosuppressive agent [24], while the fifth hit, glimepiride, is an antidiabetic agent [25]. Hydrocortisone succinate formed seven van der Waals, one conventional H-bond, one charge, and one pi interactions with the target. On the other hand, the interaction of glimepiride with $\beta$-clamp is much simpler, it featured only six van der Waals and a conventional hydrogen bond. Glimepiride is a second-generation sulfonylurea (SU) drug used for the management of type 2 diabetes mellitus (T2DM) [26]. It works by blocking ATP-sensitive potassium channels (KATP channels) that causes depolarization of the beta cells, and thereby 
stimulating the secretion of insulin granules from pancreatic islet beta cells $[25,26]$. Although these drugs were originally developed for different indications, this in silico drug repurposing effort suggests that they can be developed as antibiotic against $H$. pylori.

\section{ADME-Tox}

To become a good drug candidate, a compound should have, among others, optimal aqueous solubility, good human intestinal absorption, low hepatoxicity, low $(<90 \%)$ plasma protein binding (PPB) potential, and does not inhibit cytochrome P450 (CYP2D6 non-inhibitor). Additionally, it should be non-mutagenic, noncarcinogenic, non-toxic to developing embryo or fetus, and biodegrad- able. Table 2 shows that the top five compounds are all non-inhibitors of cytochrome P540 (CYP2D6) and exhibited satisfactory solubility. Nevertheless, the top 3 have poor intestinal absorption and three out of five are toxic to the liver and have high plasma protein binding ability.

The top three compounds, namely, benfotiamine, raltitrexed, and netarsudil, are predicted to be non-carcinogenic and non-mutagenic save netarsudil. Except for raltitrexed, the top five drugs may not be administered to pregnant women (i.e. positive for developmental toxicity). Incidentally, netarsudil is the only hit that gives positive result to Ames mutagenicity. Lastly, only netarsudil and glimepiride are expected to be nonbiodegradable under aerobic conditions.

\begin{tabular}{|l|c|c|c|c|c|}
\hline \multicolumn{1}{|c|}{ Compound } & Absorption & Solubility & $\begin{array}{c}\text { CYP2D6 } \\
\text { Binding }\end{array}$ & Hepatotoxicity & $\begin{array}{c}\text { Plasma Protein Binding } \\
\text { (PPB) }\end{array}$ \\
\hline Benfotiamine & Poor absorption & Yes, low solubility & Non-inhibitor & Non-toxic & $>95 \%$ \\
\hline Raltitrexed & Poor absorption & Yes, optimal solubility & Non-inhibitor & Toxic & $<90 \%$ \\
\hline Netarsudil & Very poor absorption & Yes, optimal solubility & Non-inhibitor & Toxic & $>90 \%$ \\
\hline $\begin{array}{l}\text { Hydrocortisone } \\
\text { succinate }\end{array}$ & Moderate absorption & Yes, optimal solubility & Non-inhibitor & Non-toxic & $<90 \%$ \\
\hline Glimepiride & Good absorption & Yes, good solubility & Non-inhibitor & Toxic & $>95 \%$ \\
\hline
\end{tabular}

Table 2: Predicted ADMET properties of the original top hits.

\begin{tabular}{|l|c|c|c|c|}
\hline \multicolumn{1}{|c|}{ Compound } & Carcinogenicity & Ames Mutagenicity & Developmental Toxicity Potential & Aerobic Biodegradability \\
\hline Benfotiamine & - & - & + & + \\
\hline Raltitrexed & - & - & - & + \\
\hline Netarsudil & - & + & + & - \\
\hline Hydrocortisone succinate & + & - & + & + \\
\hline Glimepiride & + & - & + & - \\
\hline
\end{tabular}

Table 3: Predicted toxicity properties of the original top hits.

\section{Conclusion}

Virtual screening and molecular docking of approved and experimental drugs against Helicobacter pylori DNA Polymerase III $\beta$-clamp were performed to identify known drugs that can be repurposed as new antibiotics. Out of more than 8000 compounds in Drug Bank, a total of 67 compounds were identified to have greater (more negative) binding energies than 5-chloroisatin, the known ligand of the drug target. The top five hits, whose binding energy values were at least 4 times larger than the reference, include benfotiamine, raltitrexed, netarsudil, hydrocortisone succinate, and glimepiride. The in silico ADME-Tox studies showed that the top hit benfotiamine possesses most of the characteristics of a good drug: non-carcenogenic, non-mutagenic, non-toxic to the liver, and non-inhibitor of cytochrome P540. However, its drawbacks include poor solubility, low intestinal absorption, and high plasma protein binding, which can be eventually addressed by employing appropriate drug delivery system. In general, the other top hit drugs also displayed rather satisfactory drug properties. Although these drugs were originally designed for other diseases, they may now be further developed as new drug candidates for the treatment of $H$. pylori infection.

\section{Acknowledgement}

We are grateful to the Enhanced Creative Work and Research Grant (ECWRG 2017-1-032) and Emerging Interdisciplinary Research (EIDR) Program of the University of the Philippines System (OVPAA-EIDR 12-001-121102) for the use of the computing facilities.

\section{Bibliography}

1. WHO (World Health Organization). Global priority list of antibiotic-resistant bacteria to guide research, discovery, and development of new antibiotics (2017). 
2. Alba C., et al. "Antibiotic resistance in Helicobacter pylori". Current Opinion in Infectious Diseases 30.5 (2017): 489-497.

3. Atherton JC and Blaser MJ "Coadaptation of Helicobacter pylori and humans: ancient history, modern implications". Journal of Clinical Investigation 119 (2009): 2475-2487.

4. $\quad$ Pandey P., et al. "Targeting the $\beta$-clamp in Helicobacter pylori with FDA-approved drugs reveals micromolar inhibition by diflunisal". FEBS Letters 591.15 (2017): 2311-2322.

5. Burkitt MD., et al. "Helicobacter pylori-induced gastric pathology: Insights from in vivo and ex vivo models". Disease Models and Mechanisms 10.2 (2017): 89-104.

6. Megraud F and Lehours P. "Helicobacter pylori detection and antimicrobial susceptibility testing". Clinical Microbiology Reviews 20 (2007): 280-322.

7. Hayner JN and Bloom LB. "The $\beta$ Sliding Clamp Closes around DNA prior to Release by the Escherichia coli Clamp Loader $\gamma$ Complex". Journal of Biological Chemistry 288.2 (2012): 11621170.

8. Bertram JG., et al. "Increased dNTP binding affinity reveals a nonprocessive role for Escherichia coli $\beta$ clamp with DNA polymerase IV". Journal of Biological Chemistry 279.32 (2004): 33047-33050.

9. Lopez de Saro FJ and O'Donnell M. "Interaction of the beta sliding clamp with MutS, ligase, and DNA polymerase I". Proceedings of the National Academy of Sciences USA 98 (2001): 8376-8380.

10. Kurz M., et al. "Interaction of the sliding clamp beta-subunit and Hda, a DnaA-related protein". Journal of Bacteriology 186 (2004): 3508-3515

11. O'Donnell M., et al. "Clamp loader structure predicts the architecture of DNA polymerase III holoenzyme and RFC". Current Biology 11.22 (2001) R935-R946.

12. Yin MB., et al. "Cyclin E-cdk2 activation is associated with cell cycle arrest and inhibition of DNA replication induced by the thymidylate synthase inhibitor Tomudex". Experimental Cell Research 25 247.1 (1999): 189-199.

13. Pandey P., et al. "Structural insight into beta-clamp and its interaction with DNA ligase in Helicobacter pylori". Scientific Reports 6 (2016): 31181.

14. Sliwoski G., et al. "Computational methods in drug discovery". Pharmacology Review 66.1 (2013): 334-395.

15. Ou-Yang SS., et al. "Computational Drug Discovery". Acta Pharmacologica Sinica 33.9 (2012): 1131-1140.
16. Billones JB., et al. "In silico discovery and in vitro activity of inhibitors against Mycobacterium tuberculosis 7,8-diaminopelargonic acid synthase (Mtb BioA)". Drug Design, Development and Therapy 11 (2017): 563-574.

17. Billones JB., et al. "Toward antituberculosis drugs: in silico screening of synthetic compounds against Mycobacterium tuberculosis l,d-transpeptidase 2". Drug Design, Development and Therapy 10 (2016): 1147-1157.

18. Yang CTM and Billones JB. "Towards Antituberculosis Drugs: Molecular Docking of Curcumin and Its Analogues to Pantothenate Synthetase". Philippine Journal of Science 141.2 (2012): 187-196.

19. Uy VCC and Billones JB. "Towards Antituberculosis Drugs: Virtual Screening for Potential Inhibitors of Pantothenate Synthetase of Mycobacterium tuberculosis". Philippine Science Letters 5.2 (2012): 122-130.

20. Pandey P., et al. "Screening of E. coli beta-clamp inhibitors revealed that few inhibit Helicobacter pylori more effectively: structural and functional characterization". Antibiotics 7.1 (2018): 5 .

21. Ma DL., et al. "Molecular docking for virtual screening of natural product databases". Chemical Science 2.9 (2011): 16561665.

22. McCarty MF., et al. "Targeting oxidant stress as a strategy for preventing vascular complications of diabetes and metabolic syndrome". In: Pasupuleti VK and Anderson JW. (Eds.). Nutraceuticals, glycemic health and type 2 diabetes (1st ed.). Ames, Iowa: Wiley-Blackwell/IFT Press. p213, (2008).

23. Lin CW., et al. "Discovery and Preclinical Development of Netarsudil, a Novel Ocular Hypotensive Agent for the Treatment of Glaucoma". Journal of Ocular Pharmacology and Therapeutics 34 (2017): 40-51.

24. Elks J. "The dictionary of drugs: chemical data: chemical data, structures and bibliographies". Springer (2014).

25. Massi-Benedetti M. "Glimepiride in type 2 diabetes mellitus: a review of the worldwide therapeutic experience". Clinical Therapeutics 25.3 (2003): 799-816.

26. Sola D., et al. "Sulfonylureas and their use in clinical practice". Archives of Medical Science 11.4 (2015): 840-848.

\section{Volume 3 Issue 12 December 2019 (C) All rights are reserved by Prince Messiah G Tan and Junie B Billones.}

